\title{
Fundus changes in mesangiocapillary glomerulonephritis type II: vitreous fluorophotometry
}

\author{
M F RAINES,' J DUVALL-YOUNG, ' AND C D SHORT \\ From the 'Manchester Royal Eye Hospital, 'Department of Ophthalmology, University of Manchester, and \\ ${ }^{3}$ Department of Renal Medicine, University of Manchester
}

SUMMARY We have described a complex abnormality of retinal pigment epithelium, Bruch's membrane, and choriocapillaris in mesangiocapillary glomerulonephritis (MCGN) type II. Patients with MCGN type II were examined by vitreous fluorophotometry which reveals that there is a breakdown of the blood retinal barrier (BRB) in those patients with the typical fundus lesions. The function of this barrier was calculated as a penetration ratio and was statistically greater in these patients when compared with a group of $(a)$ normal persons, $(b)$ patients with drusen, and $(c)$ patients with other forms of glomerulonephritis.

We have described marked changes in the complex of retinal pigment epithelium, Bruch's membrane, and choriocapillaris in patients with mesangiocapillary glomerulonephritis (MCGN) type II, by histopathological examination' and by clinical examination, including fluorescein angiography. 'The characteristic abnormality clinically has the appearance of widespread drusen but histopathologically was shown to be a deposit in the choriocapillaris and Bruch's membrane, similar to the diagnostically electron dense deposit seen in the renal glomerulus by ultrastructural examination.

We examined a group of patients with MCGN type II by vitreous fluorophotometry and compared them with a group of normal persons, a group of patients with other forms of glomerulonephritis, and a group of patients with degenerative drusen.

\section{Patients and methods}

Of the group of patients previously studied' with glomerulonephritis and/or partial lipodystrophy (PLD), 15 were also examined by vitreous fluorophotometry. Thirteen of these patients had biopsy proved renal disease: four had MCGN type II, four had MCGN type I, and five had membranous glomerulonephritis. A further two patients had PLD, which is frequently associated with MCGN type II. One of these latter patients had no evidence of Correspondence to M F Raines. FRCS Ed, Birmingham and Midland Eye Hospital, Church Street. Birmingham B3 2NS. glomerular disease despite many years of observation, with normal serum creatinine values and no proteinuria. The other had reached end stage renal failure, no biopsy was available but the presumed diagnosis was MCGN type II.

The classification of the renal lesion was withheld from the ophthalmologists who examined the patients by routine clinical methods.

All patients undergoing vitreous fluorophotometry were normotensive at the time of examination and had been for several months with or without treatment. None were diabetic, a condition known to affect the fluorophotometric readings. Careful indirect ophthalmoscopy and slit-lamp biomicroscopy excluded other conditions such as posterior vitreous detachment, which may also affect the readings.

With written consent vitreous fluorophotometry was performed with the Fluorotron Master (Coherent, Palo Alto, California) by standard recognised techniques. ${ }^{45}$ Both eyes were dilated with tropicamide $1 \%$ and phenylephrine $10 \%$ to attain maximum dilatation and cycloplegia. A baseline background scan was performed on each cye to assess the natural fluorescence of the eyc. Sodium fluorescein was injected intravenously at the antecubital fossa in a dose of $14 \mathrm{mg} / \mathrm{kg}$ body weight. Standard fluorescein angiography was then performed. Between 3 and 5 minutes after injection a further scan, termed the bolus scan, was recorded on each eye to account for any chorioretinal spread function that may occur. This spread function is a 
result of fluorescein in the choroid-retina influencing the readings taken in the vitreous. The bolus scan may be used in the final calculations to minimise this influence. A final measurement scan was read at 60 minutes after injection.

Venous blood, $10 \mathrm{ml}$, was drawn from the opposite arm to the fluorescein injection, between 5 and 10 minutes after injection, and also at the end of the examination. Exact times of scans and venepuncture after fluorescein injection were recorded. Plasma fluorescence was measured by means of the special cuvettes and the plasma adapter supplied with the Fluorotron. The plasma values were incorporated into a recognised computer algorithm to attain the fluorescein plasma integral.

For analysis we used the ARVO 85 (Coherent) program. The preinjection scan was subtracted from the measurement scan as only low levels of fluorescein leakage were identified. This manoeuvre minimises the contribution of natural fluorescence, which comes predominantly from the lens. The average value of fluorescence at a distance between $2 \mathrm{~mm}$ and $4 \mathrm{~mm}$ anterior to the retina was recorded. This has been shown to be relatively free of interference from the chorioretinal spread function.5 A final posterior vitreous penetration ratio (PR) for the eye was obtained from this finding."

Some patients were examined on more than one occasion, in which case the penetration ratios of the different recordings were averaged. Results from these patients were then compared with penetration ratios obtained from a group of normal individuals (49 eyes from 35 patients) and also from a group of patients with clinically and fluorescein angiographically proved drusen ( 11 eyes from 11 patients).

\section{Results}

The four patients with MCGN type II had clinical and fluorescein angiographically demonstrable deposits in the fundus in both eyes." The one patient with PLD, end stage renal failure, and presumed MCGN type II had penetration ratios (average $\mathrm{PR}=1.514$ ) within the normal range despite having clinical and fluorescein angiographically proved deposits in the fundus. The other patient with PLD but without detectable renal disease had abnormally high penetration ratios $(P R=3.41)$ despite having a clinically normal fundus. The mean penetration ratio values are shown in Table 1.

Normal methods of analysis rely on observations being independent, which they are patently not if measurements are performed on both eyes of the same individual. The method of Rosner, ${ }^{7}$ which adjusts for this lack of independence, was employed by means of the GLIM statistical package. ${ }^{k}$ This showed that age had an insignificant negative effect on the values $(p=0 \cdot 116)$. With this small effect controlled for and the correlation between eyes taken into account, the findings were that the PRs in patients with MCGN type II were significantly greater than those of all other patients $(p=0 \cdot() 18)$. The PRs of the patients with drusen were not significantly different from those of the normal control patients. The PRs of the remaining renal patients were lower than those of the normal controls $(p=0 \cdot() 33)$.

All patients had plasma albumin concentrations within normal limits. There was no significant difference between the plasma albumin levels in type II MCGN and the remaining types of glomerulonephritis. Similarly, there was no significant difference in the 24-hour urinary protein in these two groups.

\section{Discussion}

We have previously noted that a comparison between the anatomy of the glomerulus and the complex of the choriocapillaris, Bruch's membrane, and retinal pigment epithelium can be made and have documented the ocular changes found in mesangiocapillary glomerulonephritis (MCGN) type II - that is, deposits in the choriocapillaris and Bruch's membrane, having similar histopathological characteristics to those in the glomerulus, but with the clinical appearance of drusen. ' 2 Our findings with vitreous fluorophotometry show that there is breakdown of the blood retinal barrier in patients with MCGN type II. All our patients were unaware of any ocular lesion.

Vitreous fluorophotometry has been shown to be a sensitive and quantifiable method of assessing the blood retinal barrier, ${ }^{10}$ which is found at the tight junctions of the retinal pigment epithelial cells and the endothelial cells of the retinal capillaries." I2

Vitreous fluorophotometry has been recorded in many ocular conditions, including diabetic ${ }^{641013-16}$ and hypertensive ${ }^{17-19}$ retinopathies, pars planitis, ${ }^{2 "}$ and retinitis pigmentosa. ${ }^{31-25}$ In these conditions the BRB breaks down, as shown by increased leakage of fluorescein into the vitreous. The degree of

Table 1 Posterior vitreous penetration ratios

\begin{tabular}{llll}
\hline $\begin{array}{l}\text { Type } \\
\text { No of } \\
\text { patients }\end{array}$ & $\begin{array}{l}\text { No of } \\
\text { eyes }\end{array}$ & $\begin{array}{l}\text { Mean penetration } \\
\text { ratios } \\
\times 10 \mathrm{~N} / \text { minute } \\
(S D)\end{array}$ \\
\hline MCGN II & 5 & 10 & $3 \cdot(099(0 \cdot 946)$ \\
Drusen & 11 & 11 & $2 \cdot 280(0 \cdot 615)$ \\
Normals & 35 & 49 & $2 \cdot 313(0 \cdot 676)$ \\
Other renal patients & 10 & 20 & $1 \cdot 818(0 \cdot 762)$ \\
\hline
\end{tabular}


fluorescence detected in the vitreous is related to the extent of chorioretinal disease, such that increased penetration ratios are found with increased severity of, for example, diabetic retinopathy. If we postulate that the deposits found in the eyes of patients with MCGN type II are similar to drusen, then the overlying pigment epithelium may atrophy with time, as is seen with drusen..$^{2 h}$ With this atrophy there may exist breakdown of the $\mathrm{BRB}$, which may explain the findings in our patients.

One of our patients with PLD had been followed for many years without evidence of renal impairment: her serum creatinine had always been normal and there was no proteinuria. However, her vitreous fluorophotometry readings and penetration ratios were abnormally high $(\mathrm{PR}=3.41)$ and were reproducible on different occasions. Fluorescein angiography gave normal results. We are unable to explain this high reading, though it may represent a subclinical phenomenon, as it is known that some patients with PLD have a histological renal lesion which is clinically undetectable. ${ }^{77}$ Likewise this patient may have had ocular disease which was undetectable by conventional methods of examination, including fluorescein angiography. We also found vitreous fluorophotometric readings within normal limits in one patient who did have clinical and fluorescein angiographically proved chorioretinal deposits $(\mathrm{PR}=1.514)$. The lesions seen, however, were not as florid as those of the other renal patients and in particular were not extensive at the macula (Fig. 1).

None of our patients had other detectable ocular lesions. Systemic hypertension can affect the fluorophotometry readings, ${ }^{17-19}$ but none of our patients had signs of hypertensive retinopathy.

Our group of patients with drusen did not show penetration ratios of the same magnitude as our patients with MCGN type II. This can be explained by the fact that the drusen observed (Fig. 2) were not as widespread as the deposits found in the patients with MCGN type II (Fig. 3). In a recent report of a group of patients with drusen fluorophotometric readings were found to be within normal limits, but the extent of the drusen was not specified. ${ }^{2 x}$ Owing to the widespread nature of the deposit we describe, it cannot be compared directly with drusen. The findings, however, indicate that deposits in Bruch's membrane cause retinal pigment epithelial dysfunction. This has never been previously demonstrated. That the dysfunction is present at the cellular level or at the cell junctions themselves is impossible to differentiate.

We propose that there is retinal pigment epithelial dysfunction in MCGN type II as shown by the breakdown of the blood retinal barrier, and this is

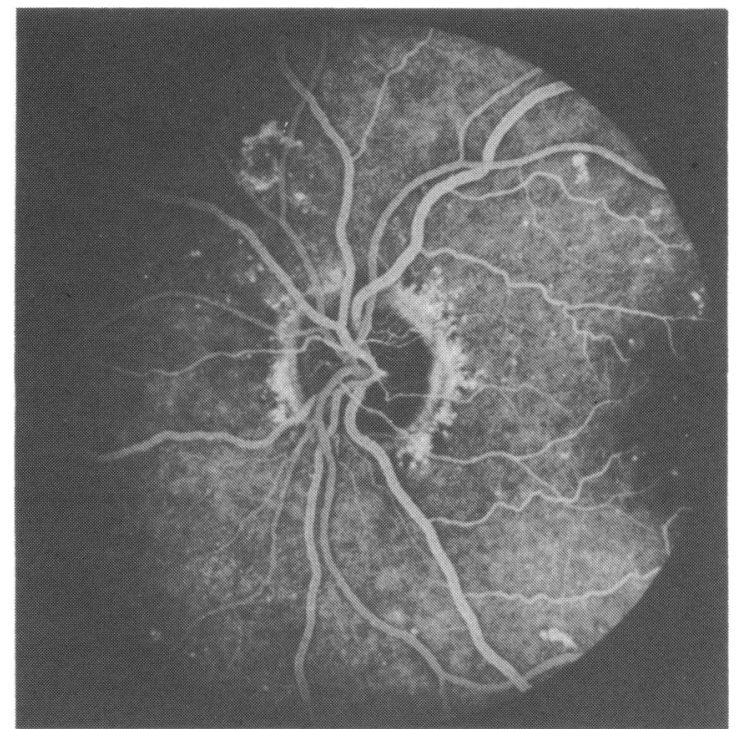

Fig. 1 Fluorescein angiogram of patient with MCGN II showing minimal chorioretinal deposits particularly at the macula, and normal penetration ratios.

likely to be a result of the deposits interfering with the retinal pigment epithelial layer.

The fluorophotometric findings in diabetics without retinopathy are controversial, ${ }^{641013-16}$ but if an abnormality does exist our findings support the possibility of an asymptomatic retinal pigment epithelial dysfunction. Because of the similarity

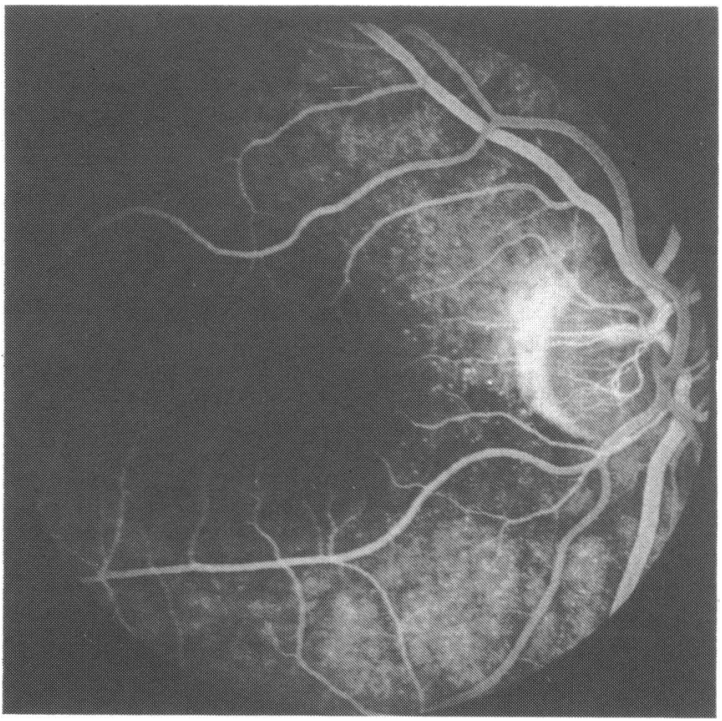

Fig. 2 Fluorescein angiogram showing extent of typical patient with drusen. 


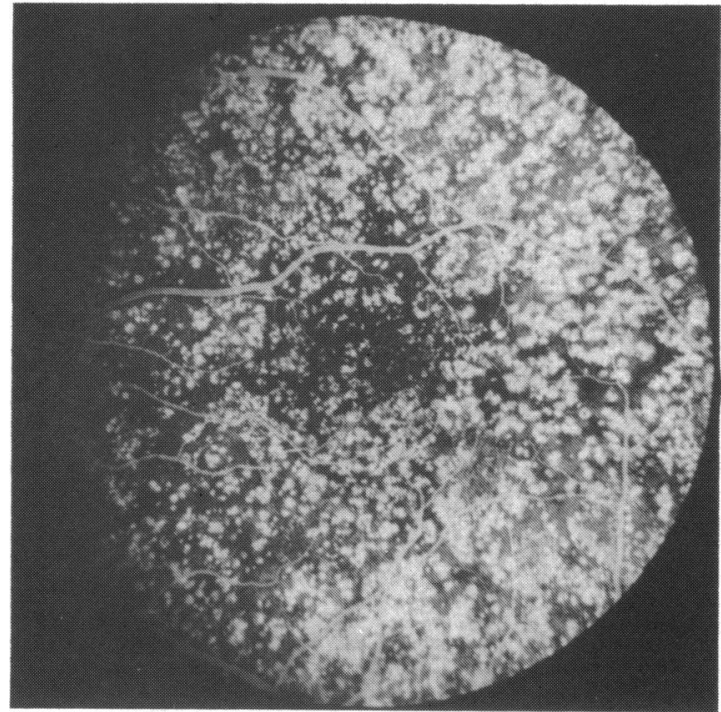

Fig. 3 Fluorescein angiogram showing typical extent of chorioretinal deposits at the macula in patient with MCGN II and raised penetration ratios.

between the anatomy of the glomerulus and the complex of choriocapillaris, Bruch's membrane and retinal pigment epithelium it would be helpful to explore eye and renal function in parallel in other disease processes.

We are grateful for advice from Mr David Shaw, medical statistician, University of Birmingham Medical School.

\section{References}

1 Duvall-Young J, MacDonald MK, McKechnie NM. Fundus changes in (type II) mesangiocapillary glomerulonephritis simulating drusen: a histopathological report. Br J Ophthalmol 1989; 73: 297-302.

2 Duvall-Young J, Short CD, Raines MF, Gokal R, Lawler W. Fundus changes in mesangiocapillary glomerulonephritis type II: clinical and fluorescein angiographic findings. $\mathrm{Br} J$ Ophthalmol 1989; 73: 900-6.

3 Darmady EM, Maliver AS. Renal pathology. London: Butterworths, 1980.

4 Zeimer RC, Blair NP, Cunha-Vaz JG. Vitreous fluorophotometry for clinical research. I. Description and evaluation of a new fluorophotometer. Arch Ophthalmol 1983; 101: 1753-6.

5 Zeimer RC, Blair NP, Cuhna-Vaz JG. Vitreous fluorophotometry for clinical research. II. Method of data acquisition and processing. Arch Ophthalmol 1983; 101: 1757-61.

6 Cunha-Vaz JG, Gray JR, Zeimer RC, Mota MC, Ishimota BM, Leite $E$. Characterization of the early stages of diabetic retinopathy by vitreous fluorophotometry. Diabetes $1985 ; 34$ : 53-9.

7 Rosner B. Multivariate methods in ophthalmology with applications to other paired data situations. Biometrics 1984; 40: 1025-35.
8 Baker RJ, Nelder JA. The Glim system. Release 3. Oxford: Numerical Algorithms Group, 1985.

9 Cunha-Vaz JG, Faria De Abreu JR, Campos AJ, Figo GM. Early breakdown of the blood retinal barrier in diabetes. $\mathrm{Br} \mathrm{J}$ Ophthalmol 1975: 59: 649-56.

10 Waltman SR, Oestrich CA, Krupin T, et al. Quantitative vitreous fluorophotometry. A sensitive technique for measuring early breakdown of the blood retinal barrier in young diabetic patients. Diabetes 1978; 27: 85-7.

11 Shiose Y. Electron microscope studies on blood retinal and blood aqueous barriers. Jpn J Ophthalmol 1970; 14: 73-87.

12 Shakib M, Cunha-Vaz JG. Studies on the permeability of the blood retinal barrier. IV. Junctional complexes of the retinal vessels and their role in the permeability of the blood retinal barrier. Exp Eye Res 1966; 5: 229-34.

13 Cunha-Vaz JG, Goldberg MF, Vygantas C, Noth J. Early detection of retinal involvement in diabetes by vitreous fluorophotometry. Ophthalmology 1979: 86: 262-75.

14 Krupin T, Waltman SR, Oestrich C, et al. Vitreous fluorophotometry in juvenile onset diabetes mellitus. Arch Ophthalmol 1978; 96: $812-4$.

15 Prager TC, Chu HH, Garcia CA, et al. The use of vitreous fluorophotometry to distinguish between diabetics with and without observable retinopathy: effect of vitreous abnormalities on the measurement. Invest Ophthalmol Vis Sci 1983; 24: 57-65.

16 Krogsaa B, Lund-Anderson H, Mehisen J, Sestoft L, Larsen J. The blood retinal barrier permeabiity in diabetic patients. Acta Ophthalmol (Kbh) 1981; 59: 689-94.

17 Krogsaa B, Lund-Andersen H, Parving HH, Bjaeldager P. The blood retinal barrier permeability in essential hypertension. Acta Ophthalmol (Khh) 1983; 61: 541-4.

18 Dutton JJ, Krupin T, Waltman SR, Koloms BA, Becker B. Fluorophotometry and blood ocular barrier in experimental systemic hypertension. Arch Ophthalmol 1980; 98: 731-3.

19 Jampol LM, White S, Cunha-Vaz J. Vitreous fluorophotometry in patients with hypertension. Arch Ophthalmol 1983: 101: $888-90$.

20 Mahlberg PA, Cunha-Vaz JG, Tessler HH. Vitreous fluorophotometry in pars planitis. Am J Ophthalmol 1983; 95: 189-96.

21 Mallick KS, Zeimer RC, Fishman GA, Blair NP, Anderson RJ. Transport of fluorescein in the ocular posterior segment in retinitis pigmentosa. Arch Ophthalmol 1984; 102: 691-6.

22 Fishman GA, Cunha-Vaz J, Salzano T. Vitreous fluorophotometry in patients with retinitis pigmentosa. Arch Ophthalmol 1981 99: 1202-7.

23 Gieser DK, Fishman GA, Cuhna-Vaz J. X-linked recessive retinitis pigmentosa and vitreous fluorophotometry. A study of female heterozygotes. Arch Ophthalmol 1980; 98: 307-10.

24 Fishman GA, Cunha-Vaz JG. Carriers of X-linked retinitis pigmentosa: investigation by vitreous fluorophotometry. Int Ophthalmol 1981: 4: 37-44.

25 Travassos A, Fishman G, Cunha-Vaz JG. Vitreous fluorophotometry studies in retinitis pigmentosa. Graefes Arch Clin Exp Ophthalmol 1985; 222: 237-40.

26 Sarks SH. Drusen and their relationship to senile macular degeneration. Aust NZ J Ophthalmol 1980; 8: 117-30.

27 Bennett WM, Bardana ES, Wuepper K, et al. Partial lipodystrophy, C3 nephritic factor and clinically inapparent mesangiocapillary glomerulonephritis. Am J Med 1977; 62: 757-60.

28 Merin S, Blair NP, Tso MOM. Vitreous fluorophotometry in patients with senile macular degeneration. Invest Ophthalmol Vis Sci 1987; 28: 756-9.

Accepted for publication 16 May 1989. 